\title{
Assessment of Severity Level for Diabetic Macular Oedema Using Machine Learning Algorithms
}

\author{
S. Murugeswari' ${ }^{1}$, R. Sukanesh ${ }^{2}$ \\ ${ }^{1}$ Syed Ammal Engineering College, Ramanathapuram, India \\ ${ }^{2}$ Thiagarajar College of Engineering, Madurai, India \\ Email: murugeskabilan@gmail.com,drsukanesh@gmail.com
}

Received 28 March 2016; accepted 24 May 2016; published 27 May 2016

Copyright (C) 2016 by authors and Scientific Research Publishing Inc.

This work is licensed under the Creative Commons Attribution International License (CC BY). http://creativecommons.org/licenses/by/4.0/

c) (†) Open Access

\begin{abstract}
The macula is an imperative part present in our human visual system which is most responsible for clear and colour vision. For the people suffering from diabetes, the various parts of the body including the retina of the eye are affected. These retinal damages cause swelling and other abnormalities nearby macula. The pathologies in macula due to diabetes are called Diabetic Macular oEdema (DME). It affects patients' vision that may lead to vision loss. It can be overcome by advance identification of causes for swelling. The major causes for the swelling are neovascularization and other abnormalities occurring in the blood vessels nearby the macula. The aim of this work is to avoid vision loss by detecting the presence of abnormalities in macula in advance. The pathologies present in the abnormal images are detected by image segmentation technique viz. Fuzzy K-means algorithm. The classification is done by two different classifiers namely Cascade Neural Network and Partial Least Square which are employed to identify whether the image is normal or abnormal. The results of both the classifiers are compared with respect to classifier accuracy, sensitivity and specificity. The classifier accuracies of Cascade Neural Network and Partial Least Square are $\mathbf{9 6 . 8 4 \%}$ and $94.36 \%$, respectively. The information about the severity of the disease and the localization of pathologies are very useful to the ophthalmologist for diagnosing the disease and apply proper treatments to the patients to avoid the formation of any lesion and prevent vision loss.
\end{abstract}

\section{Keywords}

Cascade Neural Network, Diabetic Macular Oedema, Grey Level Co-Occurrence Matrix, Neovascularization, Partial Least Square Classifier 


\section{Introduction}

The people with diabetes suffer by vision loss due to the damage of retinal blood vessels in the eye. These damaged blood vessels leak out the bloods in the macula region and will lead to vision loss. This defect in the macula due to diabetes is called diabetic macular oedema. The foremost cause of diabetic macular oedma is neovascularization which is a progress of tiny and abnormal blood vessels inside the macular region. These blood vessels occur in wrong places, damage macula and cause bleeding. Another cause for macular oedema is abnormalities of blood vessels in the macula. By identifying this in advance, leakage of fluid from the vessels can be stopped. Currently, the presence of neovascularization and other abnormalities in blood vessels are detected by ophthalmologists from dilated retinal images. The dilating drops may cause irritation to the eyes and induce giddiness. This course grosses minimum of 30 minutes to work. The investigation time and effect on the patient could be reduced if the system could work based on the taken images where the pupil of the patient is not dilated. There are countless works dealt about this problem which are also reviewed here and given below.

Support vector machine is proposed to classify the severity level for different pathologies in the retina. The features are extracted from the raw images using the image processing techniques and fed to the classifier for classification. We demonstrate a sensitivity of more than $90 \%$ for the classifier with the specificity of $100 \%$ [1]. Diabetic macular oedema was identified using instantaneous amplitude and instantaneous frequency characteristics of an image. Computational complexity of this method was high [2]. Lesions were detected from low contrast, digital images with non-dilated pupils by Fuzzy C-Means (FCM) clustering [3]. The Sobel edge detector is combined with threshold to produce best qualitative segmentation for detecting choroidal neovascularization from retinal fluorescein angiograms in exudative age-related macular degeneration [4]. The motion pattern technique is introduced to detect abnormalities in macula. Two simple single class classifiers were also considered in this work for abnormality detection. A symmetry measure was used for assessing the degree of abnormality of an image as moderate or severe risk [5].

Multiple view retinal fundus images are used for the detection and quantitative measurement of disease. All available views were registered using non-morphological sparse features. Finally, optical flow was calculated for all the images and combined to build a naive height map of the macula. [6]. A simple local threshold was used to evaluate the spatial density amount of the cluster with similar pixels. A measure of spatial density of the cluster of similar pixels was evaluated [7]. Two efficient approaches were proposed for automatic detection and extraction of exudates and blood vessels in ocular fundus images. In both the methods, the enhanced segments were extracted based on Spatially Weighted Fuzzy C-Means (SWFCM) clustering algorithm, which was formulated by incorporating spatial neighborhood information into the standard FCM clustering algorithm [8]. Optic disc, fovea, lesions and blood vessels were detected using Hough transform and FCM clustering technique [9]. A new approach was used for automatic detection of microaneurysms (MA) from digital color fundus images. They formulate MA detection as a problem of target detection from clutter, where the probability of occurrence of target is considerably smaller compared to the clutter. A successive rejection-based strategy is proposed to progressively lower the number of clutter responses. The processing stages are designed to reject specific classes of clutter while passing majority of true MAs, using a set of specialized features. The true positives that remain after the final rejector are assigned a score which is based on its similarity to a true MA [10].

The neural network classifier was employed to assess the severity level of the disease [11]. Support vector machines (SVM) and probabilistic neural network (PNN) were applied to classify the severity level of diabetic retinopathy [12]. GLCM was used for feature extraction and morphological operations were used for segmentation. K-Nearest Neighbours (KNN) classifier was employed to access severity level of the disease [13]. Fuzzy C-means clustering algorithm was used for segmentation and artificial neural network classifier was used for classification of abnormalities [14]. Performance of Neural Networks was compared to exudate detection with four classifiers [15].

Lesions were detected using fixed and variable thresholds and severity level was calculated using Support vector machine and Neural network with accuracy of 90.4 and 93.4 respectively [16]. K-means algorithm was used to segment the affected area [17]. Lesions were classified based on the splat feature classification method by extracting features like spatial location, colour [18]. The neural network was proposed to detect abnormalities such as, microneurysms, haemorrhages and exudates [19]. In this paper the DME is investigated by using image processing algorithms. FKM segmentation algorithm is used to detect the pathologies nearby macula. CNN and PLS classifiers were used to classify whether the input image is normal or moderately affected or severely af- 
fected image. The feature extraction is done by GLCM.

\section{Materials and Methods}

In view of the above stated reviews, in this proposed work, non-dilated retinal images are fed as input to the pre-processing stage. It corrects the problem of illumination variation that occurred during image acquisition. The pre-processing stage involves colour space conversion, image restoration and enhancement. Fuzzy K-Means clustering technique is used to segment the pathologies nearby macula. To classify the severity level of the macular oedema disease CNN and PLS classifier algorithms are used and their results are compared with their performance parameters. The retinal image is shown in Figure 1. The proposed methodology in this paper is shown in Figure 2.

Before segmentation, the images can be applied to pre-processing methods to extract some specific piece of information.

\subsection{Preprocessing}

The pre-processing method involves colour space conversion, image restoration by median filtering and image enhancement by Contrast Limited Adaptive Histogram Equalization. The result of colour space conversion is shown in Figure 3 and the enhanced image is shown in Figure 4.

This enhanced image is given as the input to the segmentation process to segment the affected area (Abnormalities). It is essential to detect the localization of pathologies with respect to macula.

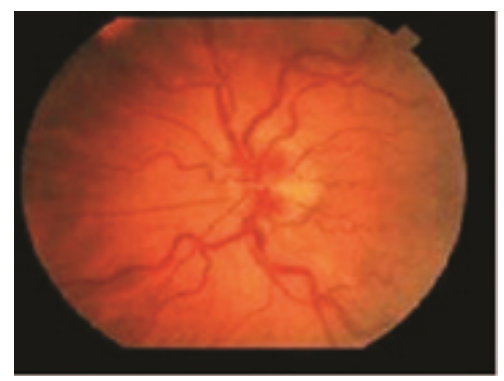

Figure 1. Retinal image.

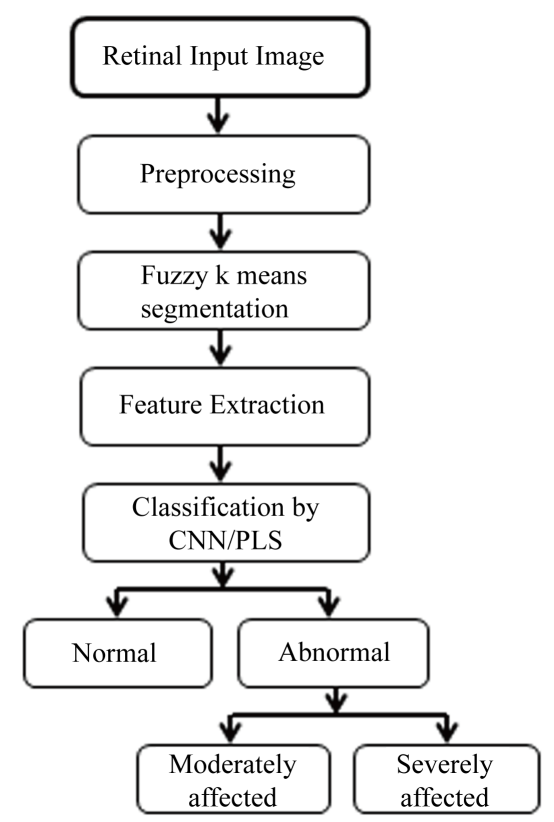

Figure 2. Proposed methodology. 


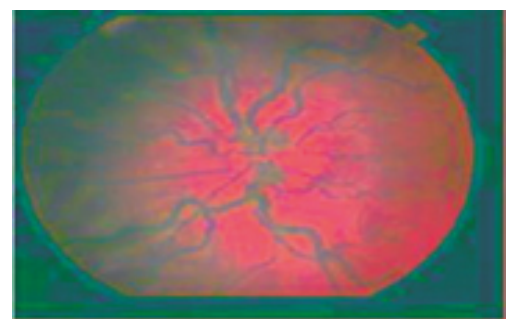

Figure 3. Colour space conversion.

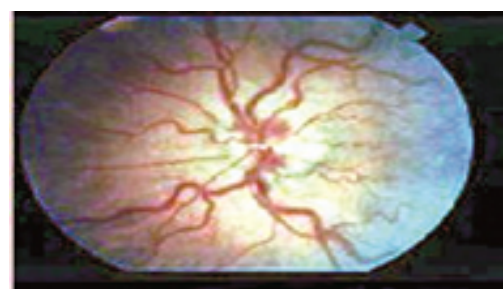

Figure 4. Enhanced mage.

\subsection{Segmentation Using Fuzzy K-Means Algorithm}

Fuzzy K-means algorithm is used for segmentation technique. K-means clustering is a technique which is usually applied to analyses data and to partition the data. The K-means clustering algorithm acquires the preferred number of different clusters and their centroids with a desired $\mathrm{K}$ number of clusters and a set of $\mathrm{k}$ initial starting points in a dataset. It detects difference between normal and abnormal region. Algorithmic steps for K-means clustering are as follows and the flow chart for $\mathrm{k}$ means segmentation algorithm is shown in Figure 5.

1. K cluster centers, either randomly or based on some investigative are picked out.

2. Each pixel in the image is assigned to the cluster that minimizes the distance between the pixel and the cluster center.

3. The cluster centers are computed by averaging all of the pixels in the cluster.

4. Steps 2 and 3 are repeated until convergence is attained (i.e. no pixels change clusters).

In this case, distance is the squared or absolute difference between a pixel and a cluster center. The difference is typically based on pixel color, intensity, texture and location, or a weighted combination of these factors. K can be selected manually, randomly, or by an investigative. This algorithm is guaranteed to converge. The quality of the solution depends on the initial set of clusters and the value of $\mathrm{K}$. This segmentation algorithm is used to identify the abnormal tiny blood vessels grown nearby the macular region and the segmented result is shown in Figure 6.

\subsection{Feature Extraction}

After segmenting the abnormal portion of an input image, Grey Level Co-occurrence Matrix extracts features from the spatial relationship of the pixel, which is important for classification of abnormalities. Different features such as correlation, homogeneity, energy, contrast, cluster prominence, thickness and Euclidean distance were calculated and these feature values are given as input to the CNN and PLS classifiers to classify the severity level for Diabetic macular Oedema.

\subsection{Classification of Severity Level of DME Using CNN and PLS}

After segmenting the abnormalities, severity of the disease is analysed using two classifiers namely Cascade Neural Network (CNN) and Partial Least Square (PLS) separately and compared with all performance parameters.

Cascade Neural Network is a feed-forward and supervised learning algorithm. Among various classifiers or NN's, the CNN is the best architecture with no back propagation. It trains and enhances new hidden units one by one building a multi-layer structure. A cascade network architecture consisting of neurons whose number of inputs, $p$, is increased from one layer to the next. At the first layer, the neuron is linked to two inputs Xi1, $\cdots, \mathrm{Xi2}$, 


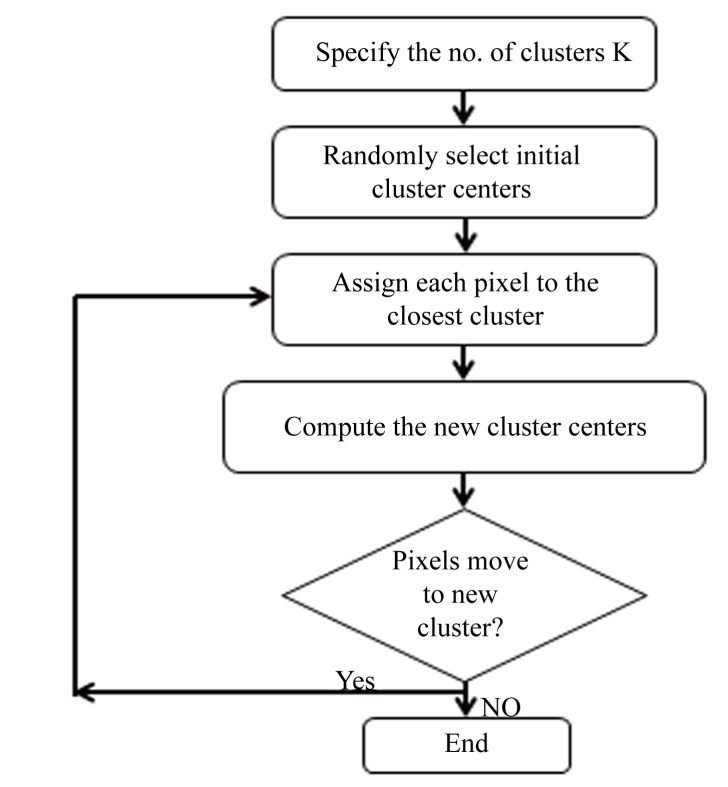

Figure 5. Flow chart for K means segmentation algorithm.

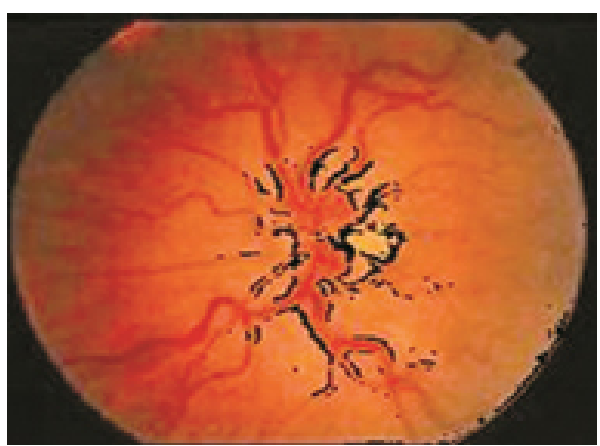

Figure 6. Segmented result.

$\mathrm{i} 1 \neq \mathrm{i} 2 \in(1, \mathrm{~m})$. Let the input Xi1 be an input for which a single-input neuron provides a minimal error. At the second layer, the new neuron is connected with the input Xi1 as well as with the output of the previous neuron. The third input of this neuron can be linked with that input which provides a maximal decrease in the output error. Each neuron at the new layer can be connected in the same manner. More formally, the neuron at the $r$ th layer has $p=r+1$ inputs. For a logistic activation function, the output $\mathrm{Zr}$ of this neuron is given in Equation (1)

$$
Z r=f(U ; W)=1 /(1+\exp (- \text { SipUiWi }))
$$

where $r$ is the number of layer, and $U=\left(U_{1}, \cdots, U_{p}\right)$ is a $p \times 1$ input vector of the $r$ th neuron.

The severity level of the disease is also assessed by using Partial Least Square Classifier. The algorithm used in this classifier is given below. [http://www.statsoft.com/Textbook/Partial-Least-Squares]

Step 1. compute $q_{h}$, the dominant eigenvector of $A_{h}^{\prime} A_{h}$

Step 2. $w_{h}=A_{h} q_{h}, \quad c_{h}=w_{h}^{\prime} M_{h} w_{h}, \quad w_{h}=w_{h} / \operatorname{sqrt}\left(c_{h}\right)$, and store $w_{h}$ into $W$ as a column

Step 3. $p_{h}=M_{h} w_{h}$, and store $p_{h}$ into $P$ as a column

Step 4. $q_{h}=A_{h}^{\prime} w_{h}$, and store $q_{h}$ into $Q$ as a column

Step 5. $v_{h}=C_{h} p_{h}$, and $v_{h}=v_{h} /\left\|v_{h}\right\|$

Step 6. $C_{h+1}=C_{h}-v_{h} v_{h}^{\prime}$ and $M_{h+1}=M_{h}-p_{h} p_{h}^{\prime}$

Step 7. $A_{h+1}=C_{h} A_{h}$.

The function of the classifier algorithm is explained in Figure 7. Every classifier determines whether the applied retinal input image is normal or abnormal based on the extracted feature and the trained images. The ab- 


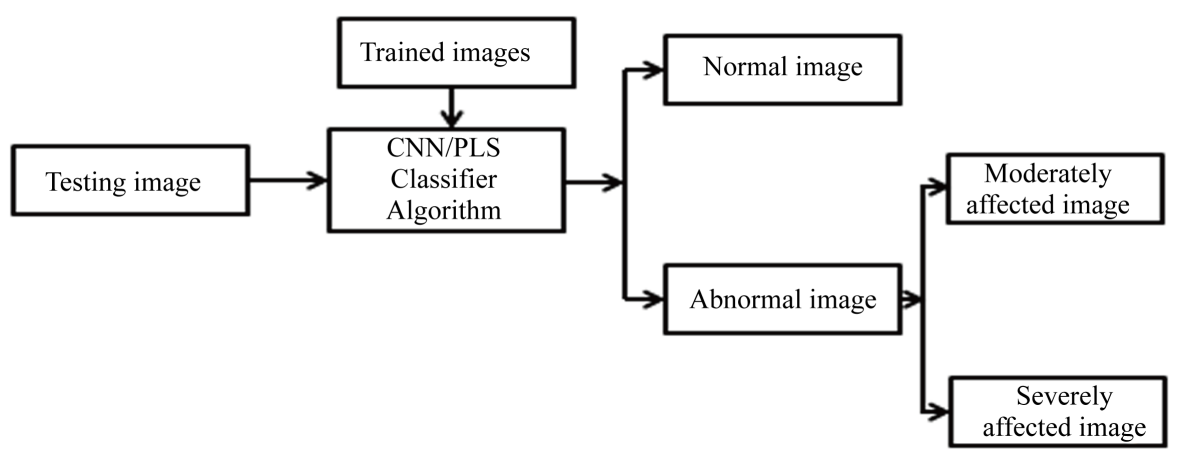

Figure 7. Function of the classifier algorithms.

normal image is further classified as moderately affected image or severely affected image.

\section{Results and Discussion}

The CNN and PLS classifiers are trained and tested with normal and abnormal images. Based on the extracted features, the classifiers are employed to classify the input images into normal or abnormal. The abnormal images are also further classified as moderate and severe to assess the severity of the disease. The data set consists of 280 non-retinal images out of which 120 images are used for training and 160 images are used for testing. Out of 120 training images, there are 40 each normal, moderately affected and severely affected images.

Out of 55 normal images tested by classifier algorithms, the CNN classifier correctly classified 54 images and 52 images were correctly classified by PLS classifier algorithm. When 50 moderately affected images were tested, 48 images were correctly classified by CNN and 47 images by PLS classifiers. Out of 55 severely affected images, the CNN and PLS classifiers correctly classified 53 and 51 images respectively. The classification accuracies and the average classifier accuracy of both the classifiers for all of the normal and abnormal images are calculated and the results are given in Table 1. The results reveal that the CNN classifier is able to identify and classify all factors which one is normal or abnormal with higher accuracy compared to PLS classifier. The tool used for obtaining the result in this research work is MATLAB R2013a.

After classifying the severity level performance of classifiers are calculated. The performance measurement parameters such as, sensitivity and specificity are measured using the formulae given below.

$$
\begin{aligned}
& \text { Sensitivity }=\mathrm{TP} /(\mathrm{TP}+\mathrm{FN}) \\
& \text { Specificity }=\mathrm{TN} /(\mathrm{TN}+\mathrm{FP})
\end{aligned}
$$

where TP, True positive $=$ correctly identified

FP, False positive $=$ incorrectly identified

$\mathrm{TN}$, True negative $=$ correctly rejected

FN, False negative $=$ incorrectly rejected

Sensitivity refers to the ability of the classifier to identify positive results and specificity related to the ability of the classifier to identify negative results. The performance parameters like sensitivity and specificity are calculated using the formulae (2) and (3). These measured parameters are presented in Table 2.

The resultant parameters such as sensitivity and specificity of classifiers are compared with those of the previously published literatures. The comparison is presented in Table 3. The CNN classifier proposed in this paper produced less sensitivity but higher specificity and accuracy than the method proposed by Sai Deepak et al. Both CNN and PLS classifiers produced better accuracy than PNN classifier proposed by Priya et al. The CNN classifier in this research work gave better sensitivity than the authors' previous work.

\section{Conclusion}

Diabetic macular oedema (DME) is a major issue for the diabetes patients as the pathologies present nearby macula make them lose their vision. The patients affected by DME can be prevented from vision loss if the disease is diagnosed in an earlier stage. To investigate the disease, the symptoms of the disease were detected. 
Table 1. Results of accuracy of CNN and PLS classifier.

\begin{tabular}{cccccccccc}
\hline \multirow{2}{*}{ Class } & \multicolumn{2}{c}{$\begin{array}{c}\text { Number of training } \\
\text { images }\end{array}$} & \multicolumn{2}{c}{$\begin{array}{c}\text { Number of testing } \\
\text { images }\end{array}$} & \multicolumn{2}{c}{$\begin{array}{c}\text { Number of images } \\
\text { correctly classified }\end{array}$} & \multicolumn{2}{c}{ Classification accuracy (\%) } \\
\cline { 2 - 9 } & CNN & PLS & CNN & PLS & CNN & PLS & CNN & PLS \\
\hline Normal & 40 & 40 & 55 & 55 & 54 & 52 & 98.18 & 96.36 \\
Abnormal (moderate) & 40 & 40 & 50 & 50 & 48 & 47 & 96 & 94 \\
Abnormal (severe) & 40 & 40 & 55 & 55 & 53 & 51 & 96.36 & 92.72 \\
& & Average classifier accuracy & & & & 96.84 & 94.36 \\
\hline
\end{tabular}

Table 2. Performance measurement of CNN and PLS classifier.

\begin{tabular}{ccc}
\hline Parameters & CNN & PLS \\
TP & 101 & 98 \\
TN & 54 & 48 \\
FP & 1 & 4 \\
FN & 2 & 96.07 \\
Sensitivity (\%) & 98.05 & 96 \\
\hline
\end{tabular}

Table 3. Comparison result of proposed method with previously published results.

\begin{tabular}{|c|c|c|c|c|c|}
\hline & $\begin{array}{l}\text { Segmentation } \\
\text { method }\end{array}$ & $\begin{array}{l}\text { Classification } \\
\text { methods }\end{array}$ & $\begin{array}{c}\text { Sensitivity } \\
(\%)\end{array}$ & $\begin{array}{c}\text { Specificity } \\
(\%)\end{array}$ & $\begin{array}{c}\text { Accuracy } \\
(\%)\end{array}$ \\
\hline $\begin{array}{c}\text { Sai Deepak et al. } \\
(2012)\end{array}$ & Motion pattern & PCA DD & 100 & 97 & 81 \\
\hline Akram et al. (2014) & $\begin{array}{c}\text { Morphological } \\
\text { operation }\end{array}$ & Hybrid classifier & 98.36 & 98.12 & 98.52 \\
\hline Priya et al. (2012) & FCM & SVM and PNN & - & - & $\begin{array}{l}97.61(\mathrm{SVM}) \\
89.60(\mathrm{PNN})\end{array}$ \\
\hline $\begin{array}{l}\text { Murugeswari and } \\
\text { Sukanesh (2014) }\end{array}$ & FCM & SVM and CNN & $\begin{array}{l}97.97(\mathrm{SVM}) \\
94.89(\mathrm{CNN})\end{array}$ & $\begin{array}{c}100(\mathrm{SVM}) \\
90.74(\mathrm{CNN})\end{array}$ & $\begin{array}{c}98(\mathrm{SVM}) \\
94.67(\mathrm{CNN})\end{array}$ \\
\hline $\begin{array}{c}\text { Mahendran and } \\
\text { Dhanasekaran (2015) }\end{array}$ & Score Computation & SVM and PNN & $\begin{array}{l}98.68(\mathrm{SVM}) \\
96.64(\mathrm{PNN})\end{array}$ & $\begin{array}{c}100(\mathrm{SVM}) \\
98.46(\mathrm{CNN})\end{array}$ & $\begin{array}{l}97.89(\mathrm{SVM}) \\
94.76(\mathrm{PNN})\end{array}$ \\
\hline Proposed work & Fuzzy K Means & CNN and PLS & $\begin{array}{c}98.05 \text { (CNN) } 96.07 \\
\text { (PLS) }\end{array}$ & $\begin{array}{c}98.18(\mathrm{CNN}) \\
96(\mathrm{PLS})\end{array}$ & $\begin{array}{c}96.84(\mathrm{CNN}) \\
94.24(\mathrm{PLS})\end{array}$ \\
\hline
\end{tabular}

The primary signs of the DME are neo-vascularization and abnormalities in blood vessels which were detected by using Fuzzy K-Means Clustering. This segmentation algorithm detects the presence and location of pathologies with respect to macula. The distance of the location of pathologies with respect to macula is useful information to find the severity of the disease. If the pathologies will be present far away from the macula, the classifier algorithm confirms the less severity. If the pathologies are very closer to the macula then it confirms the more severity and the input features needed for classification are extracted by using GLCM. The degree of severity was measured by CNN and PLS classifiers. The result of this research work gives the important information about the severity of the disease diabetic macular oedema and this work is used to detect the localization of pathologies. So, this research work is very useful to the ophthalmologist for diagnosing the disease and to apply proper treatments to the patients.

\section{References}

[1] Acharya, U.R., Chua, K.C. and Ng, E.Y.K. (2008) Application of Higher Order Spectra for the Identification of Di- 
abetes Retinopathy Stages. Journal of Medical Systems, 32, 481-488. http://dx.doi.org/10.1007/s10916-008-9154-8

[2] Agurto, C., Murray, V. and Barriga, E. (2010) Multiscale Am-Fm Methods for Diabetic Retinopathy Lesion Detection. IEEE Transactions on Medical Imaging, 29, 502-512. http://dx.doi.org/10.1109/TMI.2009.2037146

[3] Akaraspharak, U.B. and Barman, S. (2009) Automatic Exudates Detection from Nondilated Diabetic Retinopathy Retinal Images Using Fuzzy C-Means Clustering. Sensors, 9, 2148-2161. http://dx.doi.org/10.3390/s90302148

[4] Brankin, E., Muldrew, A. and Black, N. (2006) The Optimization of Thresholding Technique for the Identification of Choroidal Neo Vascular Membranes in Exudative Age-Related Macular Degeneration. 19th IEEE Symposium on Computer-Based Medical Systems, 430-435. http://dx.doi.org/10.1109/CBMS.2006.157

[5] Deepak, K. and Sivaswamy, J. (2012) Automatic Assessment of Macular Oedema from Colour Retinal Images. IEEE Transactions on Medical Imaging, 1, 766-776. http://dx.doi.org/10.1109/TMI.2011.2178856

[6] Giancardo, L., Ruggeri, A. and Chaum, E. (2011) Textureless Macula Swelling Detection with Multiple Retinal Fundus Images. IEEE Transactions on Biomedical Engineering, 58, 795-799. http://dx.doi.org/10.1109/TBME.2010.2095852

[7] Grisan, E. and Ruggeri, A. (2007) Segmentation of Candidate Dark Lesions in Fundus Images Based on Local Thresholding and Pixel Density. 2007 29th Annual International Conference of the IEEE Engineering in Medicine and Biology Society, Lyon, 22-26 August 2007, 6735-6738. http://dx.doi.org/10.1109/IEMBS.2007.4353907

[8] Kande, G.B., Subbaiah, P.V. and Savithri, T.S. (2008) Segmentation of Exudates and Optic Disc in Retinal Images. Sixth Indian Conference on Computer Vision, Graphics \& Image Processing, Bhubaneswar, 16-19 December 2008, 535- 542. http://dx.doi.org/10.1109/ICVGIP.2008.36

[9] Noronha, K., Navya, K.T. and Nayak, P.K. (2013) Hypertensive Retinopathy. International Journal of Computer Applications, 1, 7-11.

[10] Ram, K., Joshi, G.D. and Sivaswamy, J. (2011) A Successive Clutter Rejection Based Approach for Early Detection of Diabetic Retinopathy. IEEE Transactions on Biomedical Engineering, 58, 664- 673. http://dx.doi.org/10.1109/TBME.2010.2096223

[11] Krishnan, J.D.R. and Kumar, A.S. (2008) Neural Network Based Retinal Image Analysis. IEEE Conference on Image and Signal Processing, Sanya, 27-30 May 2008, 49-53.

[12] Mahendran, G. and Dhanasekaran, R. (2015) An Automatic Grading System of Severity Level for Diabetic Retinopathy using CNN Classifier. ARPN Journal of Engineering and Applied Sciences, 10, 5631-5636.

[13] Murugeswari, S. and Sukanesh, R. (2014) Detection of Diabetic Maculopathy Using KNN Algorithm. Applied Mechanics and Materials, 573, 791-796. http://dx.doi.org/10.4028/www.scientific.net/AMM.573.791

[14] Osareh, A., Mirmehdi, M., Thomas, B. and Markham, R. (2001) Automatic Recognition of Exudative Maculopathy Using Fuzzy C Means Clustering and Neural Networks. Medical Image Understanding and Analysis Conference, Bristol, 49-52.

[15] Osareh, A., Mirmehdi, M. and Thomas, B. (2002) Comparative Exudates Classification Using Support Vector Machine and Neural Networks. 5th International Conference on Medical Image Computing-Assisted Intervention, Tokyo, 25-28 September 2002, 413-420. http://dx.doi.org/10.1007/3-540-45787-9_52

[16] Reza, A.W. and Hati, C.E.S. (2008) Automatic Tracing of Optic Disc and Exudates from Colour Fundus Images Using Fixed and Variables Thresholds. Journal of Medical Systems, 33, 73-80. http://dx.doi.org/10.1007/s10916-008-9166-4

[17] Sanchez, C.I., Garcia, M., Mayo, A. and Lopez, M.I. (2009) Retinal Image Analysis Based on Mixture Models to Detect Hard Exudates. Medical Image Analysis, 13, 650-658. http://dx.doi.org/10.1016/j.media.2009.05.005

[18] Tang, L., Niemeijer, M. and Reinhardt, J.M. (2013) Splat Feature Classification with Application to Retinal Hemorrhage Detection in Fundus Images. IEEE Transactions on Medical Imaging, 32, 364-375. http://dx.doi.org/10.1109/TMI.2012.2227119

[19] Usher, D., Dumsky, M., Himaga, M., et al. (2004) Automated Detection of Diabetic Retinopathy in Digital Retinal Images: A Tool for Diabetic Retinopathy Screening. Diabetic Medicine, 21, 84-90.

http://dx.doi.org/10.1046/j.1464-5491.2003.01085.x 